\title{
Memory-Based Quantity Discrimination in Coyotes (Canis latrans)
}

\author{
Salif Mahamane ${ }^{1_{\dagger}}$, Kevin L. Grunig ${ }^{1 \dagger},{\text { Joseph } \text { Baker }^{2} \text {, Julie K. Young }}^{1,3}$, and Kerry E. Jordan ${ }^{1}$ \\ ${ }^{1}$ Utah State University \\ ${ }^{2}$ Stanford University School of Medicine \\ ${ }^{3}$ USDA, National Wildlife Research Center, Predator Research Facility \\ ${ }^{\dagger}$ These two authors contributed equally to the manuscript and share first authorship
}

*Corresponding author (Email: salif.mahamane@aggiemail.usu.edu)

Citation - Mahamane, S., Grunig, K. L, Baker, J., Young, J. K., \& Jordan, K. E. (2014). Memory-based quantity discrimination in coyotes (Canis latrans). Animal Behavior and Cognition, 1(3), 341-351. doi: 10.12966/abc.08.09.2014

\begin{abstract}
Previous research has shown that the ratio between competing quantities of food significantly mediates coyotes' (Canis latrans) ability to choose the larger of two food options. These previous findings are consistent with predictions made by Weber's Law and indicate that coyotes possess quantity discrimination abilities that are similar to other species. Importantly, coyotes' discrimination abilities are similar to domestic dogs (Canis lupus familiaris), indicating that quantitative discrimination may remain stable throughout certain species' evolution. However, while previously shown in two domestic dogs, it is unknown whether coyotes possess the ability to discriminate visual quantities from memory. Here, we address this question by displaying different ratios of food quantities to 14 coyotes before placing the choices out of sight. The coyotes were then allowed to select one of either non-visible food quantities. Coyotes' discrimination of quantity from memory does not follow Weber's Law in this particular task. These results suggest that working memory in coyotes may not be adapted to maintain information regarding quantity as well as in domestic dogs. The likelihood of a coyote's choosing the large option increased when it was presented with difficult ratios of food options first, before it was later presented with trials using more easily discriminable ratios, and when the large option was placed on one particular side. This suggests that learning or motivation increased across trials when coyotes experienced difficult ratios first, and that location of food may have been more salient in working memory than quantity of food.
\end{abstract}

Keywords - Coyotes, Canis latrans, Quantity discrimination, Short-term memory, Working memory, Numerical cognition

For many animals, sensitivity to relative differences in quantity is adaptive. Non-verbal representations of number and other quantities are shared by a multitude of species, suggesting that a representation of quantity increases an organism's likelihood of survival. Quantitative discrimination abilities across species adhere to predictions made by Weber's Law, indicating that a similar form of representation is shared across species (e.g., Brannon \& Roitman, 2003). Thus, research elucidating quantitative cognition across species is very important.

To better understand the evolutionary history of adaptive cognitive abilities, psychologists have compared cognition across many different non-human animal species (e.g., Maclean et al., 2014). In the realm of numerical cognition, similarly to humans, parrots have the ability to select the larger of two quantities based on the magnitude of difference between the choices (Ain, Giret, Grand, Kreutzer, \& Bovet, 2009; Pepperberg, 2006). Recent studies have expanded our knowledge of many other non-human animals' cognitive capacities for numerical discrimination. Chimpanzees (Beran, McIntyre, Garland, \& 
Evans, 2013), domestic dogs (Ward \& Smuts, 2007; West \& Young, 2002), sea lions (Abramson, Hernández-Lloreda, Call, \& Colmenares, 2011), various fish species (Agrillo, Dadda, \& Bisazza, 2007; Gòmez-Laplaza \& Gerlai, 2011b; Stancher, Sovrano, Potrich, \& Vallortigara, 2013), dolphins (Kilian, Yaman, Von Fersen, \& Güntürkün, 2003), gorillas (Vonk et al., 2013), bears (Vonk \& Beran, 2012), insects (Hateren, Wait, \& Srinivasan, 1990), orangutans (Vonk, 2013; Call, 2000), horses (Uller \& Lewis, 2009), various bird species (Bogale, Kamata, Mioko, \& Sugita, 2011; Garland, Low, \& Burns, 2012; Pepperberg, 2006; Ujfalussy, Miklósi, Bugnyar, \& Kotrschal, 2014) and many others (e.g., Brannon, Jordan, \& Jones, 2010; Brannon \& Roitman, 2003; Jordan \& Brannon, 2006, 2009) are capable of identifying the larger of two quantities. Common among most of these species' quantitative abilities is an adherence to predictions made by Weber's Law: non-verbal discrimination of quantities is mediated by the ratio of the competing quantities' magnitudes (e.g., Baker, Morath, Rodzon, \& Jordan 2012; Baker, Shivik, \& Jordan, 2011; Brannon \& Roitman, 2003; Jordan \& Brannon, 2006; Xu \& Spelke, 2000).

Comparing discrimination abilities in closely related species allows for a greater understanding of the numerical cognitive adaptation. Several studies have been conducted on the quantitative abilities of canids, including coyotes (Canis latrans) and domestic dogs (Canis lupus familiaris) (Baker et al., 2012; Baker et al., 2011; MacPherson \& Roberts, 2013; Ward \& Smuts, 2007). For instance, direct comparisons between domestic dogs and non-domesticated coyotes revealed similar quantitative discrimination abilities when choosing between two simultaneously visible amounts of food, suggesting that psychophysical representations of quantity are unaffected by domestication (Baker et al., 2012). Baker and colleagues $(2011,2012)$ presented coyotes and dogs with simultaneously visible choices of different food quantities, finding that both species' abilities to choose a larger amount are mediated by ratio ${ }^{1}$.

Despite the wide range of species that show similar quantitative discrimination abilities when comparing visible amounts, less work has investigated animals' ability to quantitatively discriminate using mental representation of amounts. Evolutionarily, it may be important for animals to accurately assess quantities based on memory (e.g., Meier, Burch, \& Carbyn, 1995). For example, animals may need to recall how many potential prey they saw in a previously visited location, to assess how many friends versus foes would be involved in a potential territorial dispute, or to recall how much food they have hidden in a cache (e.g., Kitchen, 2004; McComb, Packer, \& Pusey, 1994; Phillips, Ryon, Danilchuk, \& Fentress, 1991; Wilson, Hauser, \& Wrangham, 2001). Some species have indeed been shown to discriminate quantity based on memory. Jackdaws (Corvus monedula), for instance, have been found to discriminate between relative quantities on the basis of ratio when the quantities are not visible at time of choice (Ujfalussy et al., 2014). Sea lions (Otaria flavescens) show similar abilities mediated by ratio when the quantity options are visually unavailable (Abramson et al., 2011). Beran $(2001,2004)$ offered evidence of chimpanzees' abilities to mentally combine small quantities in order to gain the larger reward. Each quantity in the comparison was never seen in its entirety, effectively forcing the chimpanzee to combine mental representations of quantity with visible quantities. Orangutans also show some evidence of a comparable ability to mentally combine different amounts to gain the larger of two quantities (Call, 2000). Likewise, dogs have been found to have such abilities. Ward and Smuts (2007) found that domestic dogs are able to discriminate between two food quantities when the quantities are visually unavailable at the time of choice. Two domestic dogs were shown several quantity comparisons. After the quantities were displayed to the animals, the quantities were subsequently covered so a simultaneous visual comparison could not be made by the dogs. Ratio significantly predicted the dogs' choosing the larger quantity. MacPherson and Roberts (2013) noted that simultaneous presentation of quantities may allow dogs to effectively compare two quantities to a greater degree than sequential presentation.

Despite the known similarities between coyotes' and domestic dogs' quantitative discrimination abilities when the options are visually present, it remains unknown whether coyotes are also able to

\footnotetext{
${ }^{1}$ It should be noted that when testing quantity discrimination in animals using pieces of food with the procedure in such studies, number of pieces of food co-varies with other quantitative variables, such as surface area and presentation time-but the prediction remains that animals' choices of any such quantity will be mediated by Weber's Law.
} 
discriminate differing visual quantities based on mental (memory) representations. ${ }^{2}$ The current study addresses this topic by presenting coyotes with different numerical ratios of food pieces, which are hidden following initial presentation. Based on the findings of Baker and colleagues (2012), which found no significant difference between domestic dogs and coyotes in the ability to discriminate visible quantities, we hypothesize that coyotes will be capable of discriminating quantity based on mental representations, and that this ability will adhere to the predictions made by Weber's Law in the same manner as domestic dogs. Specifically, coyotes will be more likely to choose the larger food amount of the two options as the ratio between the two options becomes more favorable. Utilizing a similar food choice paradigm as Baker and colleagues (2011), in which the quantities were visually displayed, we instead ask whether coyotes are able to discriminate quantities based on the memory of the quantities presented. These results will provide important information that will help delineate quantity discrimination abilities across closely related canid species.

\section{Method}

\section{Participants}

We tested fourteen coyotes housed at the USDA National Wildlife Research Center (NWRC) located in Millville, Utah. Treatment of the coyotes was in accordance with APA and APHIS guidelines and approved by the NWRC IACUC. Coyotes had continuous access to water, and were fed their normal amount of food $(600 \mathrm{~g})$ on a daily basis during the study.

\section{Apparatus}

Facilities. This facility is designed so that researchers are able to study wild coyotes in as naturalistic a setting as possible. Each subject was housed in a $1000 \mathrm{~m}^{2}$ octagon-shaped pen. Each pen contained a single coyote. Every pen has natural vegetation and continuous access to water to create a semi-naturalistic setting. Each coyote is provided a den box in which it can seek shelter.

Food and preparation. The coyote food was made of proteins, fats, and grains consistent with their normal daily rations. This concoction was rolled together into food balls that were $86 \mathrm{~g}$ each.

So that at the moment of choice the coyote could not see the two quantities of food simultaneously (but instead had to rely on its memory of food quantities previously seen), feeder blinds were constructed to house the food. Two blinds were placed into each of the 14 coyote pens 3 weeks in advance of the study to allow the coyotes to habituate to this novel stimulus. Each blind had a 6x12" piece of plywood to block the view of the coyote. The plywood was screwed together using two 6" $2 \times 4$ 's flush with the bottom of the plywood, so as to allow the $6 \times 12$ " piece of wood to stand upright and maintain stability. The 4" side of the $2 \times 4$ 's was vertical with the 2 " side on the ground to block the coyote's view from the side of the blind (Figure 1). The coyotes were fed by the experimenter out of the blinds every day for the 3 week pre-study period, to habituate the coyotes to the feeding apparatus and experimenter.

An olfactory control condition was also utilized to ensure that the coyotes were not distinguishing the quantities based on the smell of the quantities. Very divergent amounts of food balls (1:6) were placed in two 12" PVC pipes with drain caps on the ends of both pipes. Out of sight of the coyote before it could make its choice, one food ball was placed into one pipe, and six food balls were placed in the other.

\footnotetext{
${ }^{2}$ Individual coyotes in the present study were not assessed on their ability to discriminate visually present quantities; however, for a general baseline for this ability for similar coyotes housed in the same complex, please see Baker and colleagues (2011).
} 


\section{Procedure}

Condition 1: Simultaneous presentation of item-by-item sets. The purpose of this condition was to determine whether coyotes can discriminate between two quantities based on memory if both quantities are initially presented at the same time in simultaneous item by item sets. An experimenter first entered $1.5 \mathrm{~m}$ into the pen carrying a white one gallon bucket to carry the food ball amounts. This experimenter kept the coyote within the central area of the pen by walking in the same general direction as the coyote until the coyote was centered.

Once the coyote was centered, the experimenter simultaneously dropped the two food choices into the blinds, dropping the food pieces one by one at a rate of one food ball per second. The food balls were placed in the feeder to prevent the coyote from seeing them at the time of choice. It was evident the coyote had seen the food balls before the time of choice because the coyote turned its head in the experimenter's direction, at which time the experimenter started dropping the food balls into the blinds. The experimenter discontinued dropping the food balls if the coyote broke visual contact and waited until the coyote's gaze returned to the experimenter to resume dropping food balls. These blinds were placed $1 \mathrm{~m}$ from each other at a distance of $1.5 \mathrm{~m}$ into the pen. The side containing the larger portion of food was randomized and counterbalanced across trials. After all food balls were placed in blinds, the experimenter walked in a straight line backwards toward the pen's entrance and exited. The experimenter walked backwards in the same straight line to an ATV that was parked 40ft away from the entrance to the pen. The experimenter then sat on the ATV, facing the pen's entrance and placing both hands symmetrically in front of him on the rim of the feeding bucket. Due to the coyotes' possible sensitivity to visual cues and tendency to be startled by movement, the experimenter remained motionless until the choice was made. Dark sunglasses were also worn by the experimenter throughout the trials, so as to not give any visual cues to the coyotes. Coyotes were coded as making a choice if they approached the blinds while lowering their head within 12 inches of the food balls. A coyote was coded as not making a decision if they did not break that threshold within a 5-minute period. Once the coyote made its decision, the experimenter entered the pen to remove the food quantity that was not chosen, while allowing the chosen quantity to be consumed.

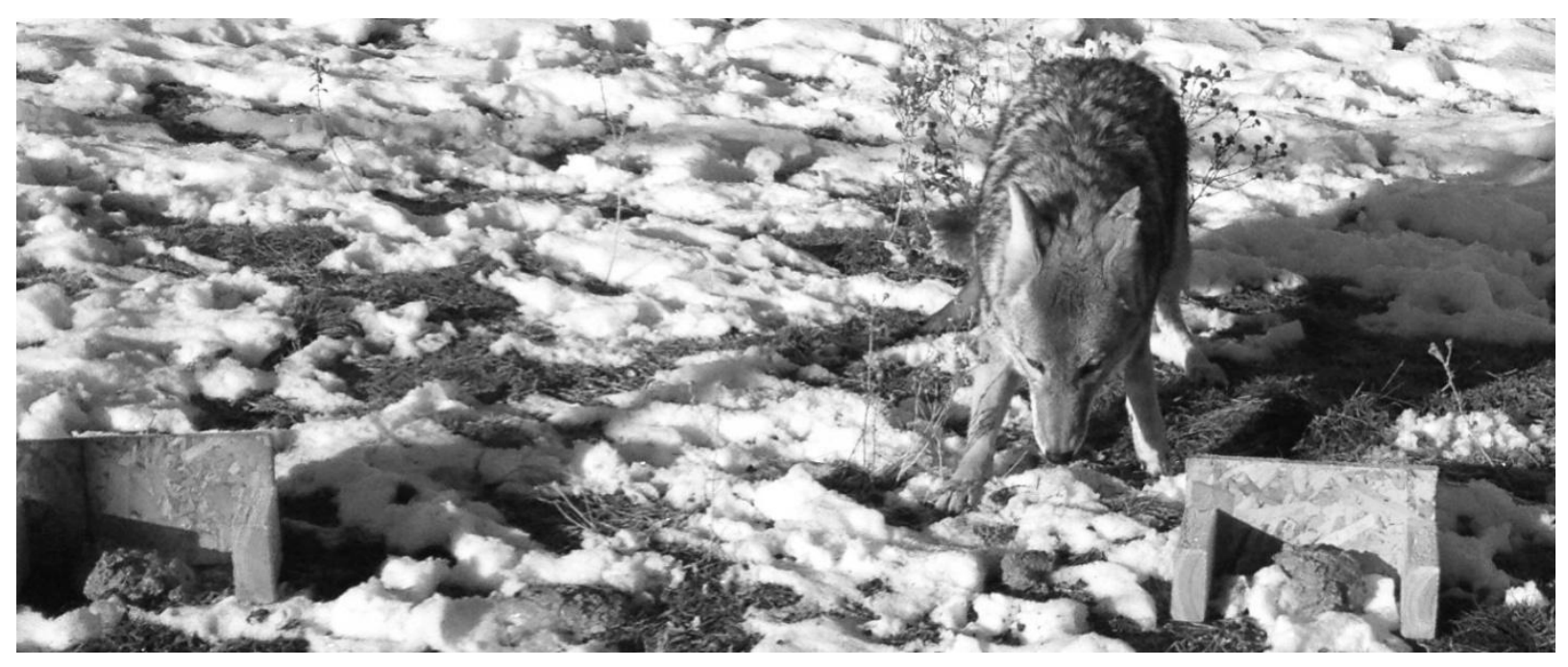

Figure 1. Coyote choosing to feed from one of the blinds.

Each coyote was tested once on each of the 9 quantity comparisons in each experimental condition (1:2, 1:3, 1:4, 1:6, 2:3, 2:4, 2:5, 3:4, \& 3:5), similar to Baker and colleagues (2011) and Ward and Smuts (2007). Just as in Baker and colleagues (2011), in order to account for possible learning effects, we randomly assigned half of the animals into one of two groups. The first group started the 
testing with the smaller ratios (i.e., 1:4), and ended with the larger ratios (i.e., 3:4). The 1:6 ratio was also used during the olfactory control trial. Each of the two 12" PVC pipes was placed in the same manner as the above procedure. The coyotes were rewarded with the amount of food balls within the chosen pipe. The unchosen portion was removed. The second group started their testing with the larger ratios and ended with the smaller ratios. Each ratio size group took one day/session to perform, so as not to satiate the coyotes. Thus, each condition was run in two sessions, one per ratio size group. Coyotes were only tested once on each quantity comparison within each condition.

Condition 2: Sequential presentation of item-by-item sets. In this condition, the experimenter dropped all relevant food balls into one blind, before dropping the remaining food balls into the other blind, to determine whether coyotes could discriminate between two quantities if the total quantities were presented in sequential item-by-item sets, rather than simultaneous item-by-item quantity presentation. The blinds were placed in the same positions as Condition 1: one meter from each other at a distance of 1.5 meters into the pen. The side that was shown first was counterbalanced and randomly chosen across trials, as was the side with the greater food quantity.

As before, the experimenter entered $1.5 \mathrm{~m}$ into the pen. The coyote was kept in the central area of the pen using the same method as Condition 1 . When the coyote was roughly centered and looking in the direction of the experimenter, the food balls were dropped into one of the food blinds. Each food ball was dropped one at a time at a rate of one food ball per second while the coyote watched. If the coyote looked away, the experimenter stopped dropping the food balls until the coyote resumed its visual contact with the experimenter. After the pre-determined number of food balls was dropped into the first blind, the experimenter dropped the other portion of food balls into the second blind using the same method. ${ }^{3}$ Exactly the same procedure of exiting the pen and waiting on the ATV for the coyote's choice as in Condition 1 was followed in Condition 2. A choice was coded in the same manner as Condition 1. After the coyote made its decision, the experimenter entered the pen to remove the other food balls that were not chosen.

Using the same nine numerical comparisons as Condition 1, each coyote was assessed on its quantity discrimination ability. Again, the 1:6 quantity comparison was also used during the olfactory control trial. Each coyote was randomly assigned to either large or small ratio size groups to start, and finished on the opposite ratio size group. Coyotes were presented with one ratio size per day/session, so as not to satiate the individual coyotes and investigate possible learning effects. The coyotes thus completed each condition in two sessions.

Each coyote underwent both conditions within a two-week period. All trials were videotaped for coding purposes.

\section{Data Analysis}

SPSS was used for statistical analysis. An alpha level of 0.05 was set. The predictions included the coyotes choosing the larger portions more often than the smaller portions, and their performance improving as the ratios increase in accordance with Weber's Law.

Binomial tests were performed to assess whether the coyotes chose the larger quantities more often than the smaller across conditions and within each condition. Binary logistic regression was used to assess the coyotes' ability to determine the larger portion by the ratio between the larger and smaller portions, large choice side, laterality (biased choosing in which an animal chooses the same side in every trial), first side placed in each trial, and whether the animal received the small or large set of ratios first (first size group). Linear regression analysis was utilized to assess the likelihood of scalar variability in coyote choice behavior. Homogeneity of variance and normality were also assessed.

\footnotetext{
${ }^{3}$ Duration of presentation (i.e., time elapsed while dropping food balls) co-varied with number and surface area as a cue to quantity, whose discrimination is also expected to be mediated by Weber's Law. Based on the current results, it is unlikely coyotes used any cues to quantity reliably to make decisions in this particular task.
} 


\section{Results}

Because there were 14 coyotes tested in nine trials per each of two conditions, there were 227 viable trials after excluding the olfactory control trials and trials for which no choice was made. ${ }^{4} \mathrm{~A}$ binomial sign test showed that across all trials and conditions, coyotes did not choose the large option significantly more often than the small option (125 trials vs. 102, respectively, $p=0.144$ ). Analyzed across both conditions, only the 1:3 (17 trials vs. 8) and the 2:4 (18 trials vs. 8) ratios showed rates of coyotes choosing the large option significantly more often than chance $(p<0.05)$. No other ratios yielded large option choices at a rate significantly different from chance when analyzed across conditions (Figure $1 \mathrm{~A})$.

Within Condition 1, there was no significant difference from chance in rate of choosing the large option across all trials (62 trials vs. 52, $p=0.399$ ). Also, for Condition 1 alone, there were no ratios within which coyotes chose the large option at a rate significantly different from chance (Figure 1B).

Within Condition 2, there was also no significant difference from chance in rate of choosing the large option across all trials (63 trials vs. 50, $p=0.259)$. However, the 1:3 ratio (10 trials vs. 2 ) and the 2:4 ratio (10 trials vs. 3 ) showed significantly greater than chance rates of choosing the large option (Figure 1C).

Regarding the olfactory control trials, in Condition 1, ten of these fourteen trials were not responded to. Of the four that were responded to, a sign test showed that the large option was never chosen $(p=0.031)$. In Condition 2, the same ten coyotes did not respond in the olfactory control trial. Of the four that did, a sign test revealed that the large option was not chosen at a rate significantly different from chance ( 1 trial vs. $3, p=0.156$ ). The results of the olfactory condition suggest that coyotes were not guided to optimal performance by olfactory cues.

To investigate whether these data replicate Baker and colleagues' (2011) findings when coyotes are presented with visible sets of differing food amounts, binary logistic regression was used to determine whether ratio significantly predicted choice while controlling for all other variables (Table 1). Because three animals exhibited laterality bias in our sample, a dummy-coded variable $(0=$ no bias, $1=$ laterality bias) was included in the regression. The predictive model was significant $\left(\chi^{2}(5)=13.112, p=0.022\right.$, Cox and Snell pseudo- $\left.R^{2}=0.056\right)$. However, ratio was not a significant predictor of the probability of making a large choice $(\beta=0.509, p>0.05)$. Large option side $(\beta=-696, p=0.017)$ and first size group $(\beta=$ $0.686, p=0.031$ ) did significantly predict the likelihood of choosing the large option. Specifically, when the large option was on the left side, coyotes were $51.1 \%$ less likely to choose the large option. Also, when coyotes were presented with the set of large ratio trials first, the likelihood of choosing the large option increased by $98.5 \%$ (For percentages of large choices made according to whether large or small ratios were shown first as a group, see Table 2). Binary logistic regressions run separately for Conditions 1 and 2 did not yield significant predictive models.

Given that large option side was a significant predictor, the regression was rerun $\left(\chi^{2}(6)=13.178\right.$, $p=0.040$, Cox and Snell pseudo- $\left.R^{2}=0.056\right)$ to include an interaction term between laterality and large option side, which showed to be non-significant $(\beta=0.181, p=0.798)$. In this regression, large option side and first size group were still significant predictors of large choice likelihood, and there were no new significant predictors.

After discovering that large option side and first size group significantly predicted the likelihood of making a large choice, it was further investigated as to whether this information could be used to understand the significantly greater than chance rate of large choice in the 1:3 and 2:4 ratios in Condition 2 and across both conditions. A binomial sign test found that in the 1:3 trials in Condition 2, right side placement was randomly determined/used at a rate significantly greater than chance (10 trials vs. $3, p=$ 0.035). No other different-from-chance rates of right side large option placement occurred in the 1:3 trials

\footnotetext{
${ }^{4}$ One coyote made no choices in Condition 1 and only responded to trials in the large ratio size group in Condition 2 . Further, in Condition 1, two 3:5 trials and one 1:6 trial were not responded to. In Condition 2, two 1:6 trials, one 3:4 trial, one 2:3 trial, one 3:5 trial, one 2:4 trial, one 1:2 trial, and one 1:4 trial were not responded to. All of these omissions constitute a total of 25 trials.
} 
across conditions or in the 2:4 trials in Condition 2 and across conditions. Rates of large ratios first were also analyzed for being different from chance, and no such differences were found for any of the relevant ratios and conditions.

Table 1

\begin{tabular}{|c|c|c|c|}
\hline Variable & $\beta$ & SE & $\operatorname{Exp}(\beta)$ \\
\hline Ratio & 0.509 & 0.756 & 1.664 \\
\hline Side & $-0.696 *$ & 0.276 & 0.499 \\
\hline Laterality & -0.024 & 0.407 & 0.976 \\
\hline First Side Placed & 0.022 & 0.171 & 0.979 \\
\hline First Ratio Size Group & $0.686 *$ & 0.317 & 1.985 \\
\hline Intercept & -0.003 & 0.496 & 0.997 \\
\hline
\end{tabular}

$* p<0.05$

Table 2

Percentage of Large Choices Made by Ratio Size Group Shown First

\begin{tabular}{cccc}
\hline Ratio & Large Ratios First & Small Ratios First & Combined \\
\hline $1: 6$ & $53.85 \%$ & $45.45 \%$ & $50 \%$ \\
$1: 4$ & $76.92 \%$ & $25 \%$ & $52 \%$ \\
$1: 3$ & $61.54 \%$ & $75 \%$ & $64 \%$ \\
$2: 5$ & $50 \%$ & $50 \%$ & $50 \%$ \\
$1: 2$ & $69.23 \%$ & $50 \%$ & $60 \%$ \\
$2: 4$ & $64.29 \%$ & $75 \%$ & $65 \%$ \\
$3: 5$ & $64.29 \%$ & $63 \%$ & $64 \%$ \\
$2: 3$ & $85.71 \%$ & $16 \%$ & $53 \%$ \\
$3: 4$ & $53.85 \%$ & $53.85 \%$ & $53 \%$ \\
\hline
\end{tabular}

Linear regression analysis across both conditions showed no significant prediction of proportion of large choices by ratio $\left(F(1,16)=0.724, p=0.407, R^{2}=0.043\right)$. Separate linear regressions for each condition also yielded non-significant predictive models (Condition $1, F(1,7)=0.001, p=0.982, R^{2}=$ 0.000 . Condition $2, F(1,7)=0.913, p=0.371, R^{2}=0.115$ ). Linear regression was also used to assess for scalar variability. Namely, ratio was regressed against variance to determine whether the latter changed as a function of the former. However, this linear regression model was also not significant across conditions $\left(F(1,16)=0.681, p=0.421, R^{2}=0.041\right)$ or for Condition $1\left(F(1,7)=0.514, p=0.496, R^{2}=0.262\right)$ and Condition $2\left(F(1,7)=0.250, p=0.632, R^{2}=0.034\right)$ separately.

Both variables--percentage of large scores and variance--were found to be non-normal; however, no transformation to the distribution was made (Li, Wong, Lamoureux, \& Wong, 2012).

\section{Discussion}

Coyotes' ability to choose the large option over the small was eliminated in this particular quantity discrimination task by their being required to rely on memory to make the optimal choice, compared with their ability to do so in previous studies with no memory component (Baker et al., 2011, 2012). In contrast to Baker and colleagues' findings (2011, 2012), and thus our predictions, coyote quantitative choice behavior in the present study did not follow Weber's Law. Ratio did not predict the proportion of large option choices by coyotes, when making such a choice based on mental representation rather than visual perception. There was no scalar variability in coyotes' choice as a function of ratio. In the present study, the likelihood of a coyote selecting the large food option was instead significantly increased by the first ratio size group presented to the coyote being large and decreased by the large option being placed on the left side.

First size group was not expected to significantly predict the likelihood of coyotes making a large choice. Coyotes were presented with the small ratios first just as often as the large ratios. This may 
indicate that learning took place across trials, in particular for the coyotes that experienced the large ratio group first. Perhaps initially seeing total greater amounts of food was more motivating to the coyotes, bolstering their performance across trials. In addition, because total number of food pieces dropped and large ratio size were confounded in the current study, coyotes received greater experience with enumerating and remembering food pieces on trials in which the ratio between food options approached 1. Future studies should remove this confound.

Large option side was also found to predict the likelihood of choosing the large option in the current study, suggesting the salience of location to coyotes when encoding or recalling details of food caches. Phillips et al. (1991) documented stereotypic movements in coyotes caching and recovering food. They hypothesized that these natural, strict action sequences enable coyotes to return to their cache sites as they are triggered when the animal enters the general location. Their research was based on the same phenomenon having been observed in captive timber wolves (Canis lupus). Thus, evolutionarily, there may not be an adaptation in coyotes for precise memory of quickly presented food quantities, but rather for recognition of location, which then triggers its corresponding action sequence to locate the cache. In the current experiment, coyotes may have developed a specific action sequence for the area containing the feeding blinds during the three-week habituation period before experimental sessions commenced. It is possible that searching the right-hand blind first is a component of this action sequence. This explanation is also supported by the findings of above-chance performance in domestic dogs for locating hidden objects up to a delay of 240s, a paradigm parallel to searching for caches (Fiset, Beaulieu, \& Landry, 2003). Such an explanation could, of course, interact with other alternative explanations. For example, coyotes may better attend to and remember food location (and perhaps amount) when caches are largeror when they make the cache themselves (e.g., perhaps they do not encode quantity into, or recall quantity from, memory when they have used only visual cues).

This conclusion does not mean that coyotes are generally insensitive to quantity, but simply that when food is not visually apparent, coyotes may search by spatial patterns rooted in automaticity as opposed to memory about quantity. Future research should incorporate 1:0 trials, as well as non-blinded trials and higher-value food rewards, to affirm these particular coyotes' ability to simply recall food location to better ground the current conclusions. Future research should also investigate whether coyotes can choose larger quantities when they are not required to track and add item-by-item sets from memory - but rather, just choose the larger of wholly presented sets based on memory of these whole sets previously seen. West and Young (2002) previously showed that domestic dogs anticipate the outcome of simple addition operations, so it is hypothesized coyotes would show some rudimentary abilities of understanding simple quantitative operations as well.

It is interesting that only two ratios, 1:3 and 2:4, showed large choice rates significantly greater than chance. In the case of the 1:3 trials in Condition 2, there was a significantly greater than chance rate of large option placement on the right side for which coyotes exhibited a bias. However, this greater-thanchance rate was not observed when assessed across both conditions, despite greater-than-chance large option choosing in the 1:3 trials across both conditions. It is uncertain as to why these ratios show significant results while the most divergent ratio, 1:6, did not. This is peculiar because many species, even taxonomically distant from coyotes, have shown discrimination in the 1:5 ratio; for example, African grey parrots (Ain et al., 2009). These findings with other species are congruent with Baker and colleagues' (2011) findings with coyotes for visible sets. Future research should investigate quantity discrimination from memory in these parrots and other species that have shown such discrimination for visible sets. On the other hand, ratio effects for quantity discrimination--specifically for sets in the small number range-aligned with Weber's Law have not always been shown in the same parrot species (Hunt, Low, \& Burns, 2008) as well as several others such as elephants (Irie-Sugimoto, Kobayashi, Sato, \& Haseqawa, 2009; Irie \& Hasegawa, 2012), angelfish (Gòmez-Laplaza \& Gerlai, 2011a), and guppies (Agrillo, Piffer, Bisazza, \& Butterworth, 2012). As noted above, further data with these particular individual coyotes in which the options are visually accessible should also be collected. If it was observed that these individual coyotes reliably selected the six food balls over the one when the options are visible, it could be argued 
that a limited working memory capacity could be responsible for the disappearance of such choices when the coyotes no longer see the two food amount options simultaneously.

While domestic dogs, close taxonomic relatives of coyotes, have shown an ability to discriminate quantity from working memory, the sample size in that experiment was very small (Ward \& Smuts, 2007). The present experiment may also be hindered by a relatively small number of trials despite the fact that the same number of animals and ratios were used as in Baker and colleagues (2011), in which ratio effects were observed with simultaneously visible quantities. These data could thus be considered preliminary in the area of coyote working memory representations of quantity.

\section{Considerations for Future Directions}

The current findings in coyotes are contrary to Ward and Smuts' (2007) finding in which two domestic dogs were able to discriminate large and small food options from memory, using a very similar paradigm. Future investigations testing possible differences between these two species that could account for this disparity, such as motivation, will be useful in determining why these closely related species differ in their ability to recall quantities from memory and use them to guide foraging decisions. It may be that a longer encoding period is necessary before the coyotes can reliably recall which blind the large option was placed in. It will also be necessary to investigate whether domestic dogs show parallel results to the current findings when a larger sample size is used, as the previous Ward and Smuts (2007) study employed only two dogs.

Some additional systematic variations to the methodology reported herein would further contribute to the comprehensiveness of our conclusions. Experimenter blindness to condition would eliminate the potential for any inadvertent cues from the experimenter to the coyote regarding the location of the large choice. Also, testing cognitive abilities across various seasons would be an important followup investigation, as it has been found that coyote foraging behavior changes with seasons (Gese, Ruff, \& Crabtree, 1996).

Various food option exposure durations should also be systematically tested, as the current experiment used brief exposure; as soon as coyotes gazed toward the food in the experimenter's hand, the experimenter began placement into the blinds. A delay between onset of the animal's attention to the food and placement into the blinds may yield different results.

Future studies could also investigate established populations of feral dogs for comparison to domestic dogs and coyotes in this regard, and determine whether different foraging requirements by these populations could help explain any divergent or similar results. Such investigations could focus on comparing the current results not only to feral dogs but also to coyotes raised from infancy (essentially domesticated) by human caretakers to better tease out potential effects of environment (specifically, human caretaking from birth).

In sum, under the particular conditions tested here, coyotes' memory-based discriminations of large and small food quantities surprisingly do not adhere to Weber's Law. Specifically, coyotes were more likely to choose the large option when they experienced trials with difficult ratios between food options first - and when the large option was placed on their right side - but overall did not choose large options significantly more often than small. Ratio did not predict the likelihood of choosing the larger choice. These results further elucidate the evolution of the adaptation of discrimination of quantity from memory, suggesting that, under the conditions tested here, working memory in coyotes may not maintain quantitative information as well as in domestic dogs.

\section{Acknowledgments}

We would like to thank USU's Office of Research and Graduate Studies for an Undergraduate Research and Creative Opportunities financial grant. We would also like to thank all of the USU Multisensory Cognition Lab personnel including Thomas Lyon, Nick Wan, and Armen Armaghanyan. 
The contributions of the USDA, National Wildlife Research Center, Predator Research Facility personnel, in Millville Utah, was essential to this study.

\section{References}

Abramson, J. Z., Hernández-Lloreda, V., Call, J., \& Colmenares, F. (2011). Relative quantity judgments in South American sea lions (Otaria flavescens). Animal Cognition, 14, 695-706. doi: 10.1007/s10071-011-0404-7

Agrillo, C., Piffer, L., Bisazza, A., \& Butterworth, B. (2012). Evidence for two numerical systems that are similar in humans and guppies. Plos ONE, 7(2), doi:10.1371/journal.pone.0031923

Ain, S., Giret, N., Grand, M., Kreutzer, M., \& Bovet, D. (2009). The discrimination of discrete and continuous amounts in African grey parrots (Psittacus etithacus). Animal Cognition, 12, 145-154. doi: 10.1007/s10071-008-0178-8

Agrillo, C., Dadda, M., \& Bisazza, A. (2007). Quantity discrimination in female mosquitofish. Animal Cognition, 10, 63-70. doi:10.1007/s10071-006-0036-5

Agrillo, C., Piffer, L., Bisazza, A., \& Butterworth, B. (2012). Evidence for two numerical systems that are similar in humans and guppies. Plos ONE, 7, 1-8. doi: 10.1371/journal.pone.0031923

Baker, J., Morath, J., Rodzon, K., \& Jordan, K. (2012). A shared system of representation governing quantity discrimination in canids. Frontiers in Psychology, 3, 387. doi: 10.3389/fpsyg.2012.00387

Baker, J. M., Shivik, J., \& Jordan, K. E. (2011). Tracking of food quantity by coyotes (Canis latrans). Behavioural Processes, 88, 72-75. doi: 10.1016/j.beproc.2011.08.006

Beran, M. J. (2001). Summation and numerousness judgments of sequentially presented sets of items by chimpanzees (Pan troglodytes). Journal of Comparative Psychology, 115,181-191. doi: 10.1037/07357036.115.2.181

Beran, M. J. (2004). Chimpanzees (Pan troglodytes) respond to nonvisible sets after one-by-one addition and removal of items. Journal of Comparative Psychology, 118, 25-36. doi: 10.1037/0735-7036.118.1.25

Beran, M. J., McIntyre, J. M., Garland, A., \& Evans, T. A. (2013). What counts for 'counting'? Chimpanzees, Pan troglodytes, respond appropriately to relevant and irrelevant information in a quantity judgment task. Animal Behaviour, 85, 987-993. doi: 10.1016/j.anbehav.2013.02.022

Bogale, B., Kamata, N., Mioko, K., \& Sugita, S. (2011). Quantity discrimination in jungle crows, Corvus macrohynchos. Animal Behaviour, 82, 635-641. doi: 10.1016/j.anbehav.2011.05.025

Brannon, E. M., Jordan, K. E., \& Jones, S. M. (2010). Behavioral signatures of numerical cognition. In M. L. Platt, A. A. Ghazanfar (Eds.), Primate neuroethology (pp. 144-159). New York: Oxford University Press. doi: 10.1093/acprof:oso/9780195326598.003.0008

Brannon, E. M., \& Roitman, J. D. (2003). Nonverbal representations of time and number in animals and human infants. In W. H. Meck (Ed.), Functional and neural mechanisms of interval timing (pp. 143-182). Boca Raton, FL: CRC Press. doi: 10.1201/9780203009574.ch6

Call, J. (2000). Estimating and operating on discrete quantities in orangutans (Pongo pygmaeus). Journal of Comparative Psychology (Washington, D.C.: 1983), 114, 136-147. doi: 10.1037W735-7036.1I4.2.I36

Fiset, S., Beaulieu, C., \& Landry, F. (2003). Duration of dogs' (Canis familiaris) working memory in search for disappearing objects. Animal Cognition, 6, 1-10.

Garland, A., Low, J., \& Burns, K. C. (2012). Large quantity discrimination by North Island robins (Petroica longipes). Animal Cognition, 15, 1129-1140. doi: 10.1007/s10071-012-0537-3

Gese, E. M., Ruff, R. L., \& Crabtree, R. L. (1996). Social and nutritional factors influencing the dispersal of resident coyotes. Animal Behaviour, 52, 1025-1043. doi: 10.1006/anbe.1996.0250

Gómez-Laplaza, L., \& Gerlai, R. (2011a). Can angelfish (Pterophyllum scalare) count? Discrimination between different shoal sizes follows Weber's law. Animal Cognition, 14, 1-9. doi: 10.1007/s10071-010-0337-6

Gómez-Laplaza, L., \& Gerlai, R. (2011b). Spontaneous discrimination of small quantities: Shoaling preferences in angelfish (Pterophyllum scalare). Animal Cognition, 14, 565-574. doi: 10.1007/s10071-011-0392-7

Hunt, S., Low, J., \& Burns, K. (2008). Adaptive numerical competency in a food-hoarding songbird. Proceedings of the Royal Society B - Biological Sciences, 275, 2373-2379. doi: 10.1098/rspb.2008.0702

Irie-Sugimoto, N., Kobayashi, T., Sato, T., \& Hasegawa, T. (2009). Relative quantity judgment by Asian elephants (Elephas maximus). Animal Cognition, 12, 193-199. doi: 10.1007/s10071-008-0185-9

Irie, N., \& Hasegawa, T. (2012). Summation by Asian elephants (Elephas maximus). Behavioral Sciences, $2,50-56$. doi: 10.3390/bs2020050 
Jordan, K. E., \& Brannon, E. M. (2006). A common representational system governed by Weber's law: Nonverbal numerical similarity judgments in 6-year-olds and rhesus macaques. Journal of Experimental Child Psychology, 95, 215-229. doi: 10.1016/j.jecp.2006.05.004

Jordan, K. E., \& Brannon, E. M. (2009). A comparative approach to understanding human numerical cognition. In B. Hood, L. Santos (Eds.), The origins of object knowledge (pp. 53-84). New York: Oxford University Press. doi: 10.1093/acprof:oso/9780199216895.001.0001

Kilian, A., Yaman, S., Von Fersen, L., \& Güntürkün, O. (2003). A bottlenose dolphin discriminates visual stimuli differing in numerosity. Learning \& Behavior, 31, 133-142. doi: 10.3758/BF03195976

Kitchen, D. M. (2004). Alpha male black howler monkey responses to loud calls: Effect of numeric odds, male companion behaviour and reproductive investment. Animal Behaviour, 67, 125 . doi: 10.1016/j.anbehav.2003.03.007

Li, X., Wong, W., Lamoureux, E., \& Wong, T. (2012). Are linear regression techniques appropriate for analysis when the dependent (outcome) variable is not normally distributed? Investigative Ophthalmology \& Visual Science, 53, 3082-3083. doi: 10.1167/iovs.12-9967

Maclean, E., Addessi, E., Amici, F., Anderson, R., Aureli, F., Baker, J., ... \& Hare, B. (2014). The evolution of selfcontrol. Proceedings of the National Academy of Sciences. www.pnas.org/cgi/doi/10.1073/pnas.1323533111

MacPherson, K., \& Roberts, W. (2013). Can dogs count?. Learning and Motivation, 44, 241-251. doi: 10.1016/j.mot.2013.04.002

McComb, K., Packer, C., \& Pusey, A. (1994). Roaring and numerical assessment in contests between groups of female lions, Panthera leo. Animal Behaviour, 47, 379.

Meier T, J., Burch J, W., \& Carbyn, N. L. (1995). Pack structure and genetic relatedness among wolf packs in a naturally-regulated population. In Carbyn, L.D., Fritss, S.H., and Seip, D.R. (Eds.), Ecology and Conservation of Wolves in a Changing World (pp. 293-302). Edmonton: Canadian Circumpolar Institute.

Pepperberg, I. M. (2006). Grey parrot numerical competence: A review. Animal Cognition, 9, 377-391. doi: 10.1007/s10071-006-0034-7

Phillips, D., Ryon, J., Danilchuk, W., \& Fentress, J. (1991). Food caching in captive coyotes: Stereotypy of action sequence and spatial distribution of cache sites. Canadian Journal of Psychology, 45, 83-91.

Stancher, G., Sovrano, V., Potrich, D., \& Vallortigara, G. (2013). Discrimination of small quantities by fish (redtail splitfin, Xenotoca eiseni). Animal Cognition, 16, 307-312. doi: 10.1007/s10071-012-0590-y

Ujfalussy, D., Miklósi, Á., Bugnyar, T., \& Kotrschal, K. (2014). Role of mental representations in quantity judgments by jackdaws (Corvus monedula). Journal of Comparative Psychology, 128, 11-20. doi: $10.1037 / \mathrm{a} 0034063$

Uller, C., \& Lewis, J. (2009). Horses (Equus caballus) select the greater of two quantities in small numerical contrasts. Animal Cognition, 12, 733-738. doi: 10.1007/s10071-009-0225-0

van Hateren, J. N., Wait, P. B., \& Srinivasan, M. V. (1990). Pattern recognition in bees: Orientation discrimination. Journal of Comparative Physiology A - Sensory, Neural, And Behavioral Physiology, 167, 649-654.

Vonk, J. (2013). Quantity matching by an orangutan (Pongo abelii). Animal Cognition, 17, 297-306.

Vonk, J., \& Beran, M. J. (2012). Bears "count" too: Quantity estimation and comparison in black bears (Ursus americanus). Animal Behaviour, 84, 231-238.

Vonk, J., Torgerson-White, L., McGuire, M., Thueme, M., Thomas, J., \& Beran, M. J. (2013). Quantity estimation and comparison in western lowland gorillas (Gorilla gorilla gorilla). Animal Cognition, 17, doi: 10.1007/s10071-013-0707-y

Ward, C., \& Smuts, B. B. (2007). Quantity-based judgments in the domestic dog (Canis lupus familiaris). Animal Cognition, 10, 71-80. doi: 10.1007/s10071-006-0042-7

West, R. E., \& Young, R. J. (2002). Do domestic dogs show any evidence of being able to count? Animal Cognition, 5, 183-186. doi: 10.1007/s10071-002-0140-0

Wilson, M. L., Hauser, M. D., \& Wrangham, R. W. (2001). Does participation in intergroup conflict depend on numerical assessment, range location, or rank for wild chimpanzees? Animal Behaviour, 61, 1203-1216. doi: 10.1006/anbe.2000.1706

Xu, F., \& Spelke, E. S. (2000). Large number discrimination in 6-month-old infants. Cognition, 74, B1-B11. 